\section{Artificial laboratories}

Michael Berry

Would-be Worlds: How Simulation is Changing the Frontiers of Science. By John L. Casti. Wiley: 1996. Pp. 242. £17.99, \$24.95.

IN his Discourse on Method, published in 1637, Descartes described how he understood the essential physics of rainbows by carrying out what we would now call a numerical simulation. At the frontier of physics in those days was Snell's law of refraction, which Descartes used to plot sun-rays through a raindrop. He discovered that the emergent rays were concentrated at an angle near $42^{\circ}$ from the direction back towards the Sun, and thereby explained the rainbow as directional focusing (an 'angular caustic'). Recently, I have been using Maxwell's laws of physics, together with a small computer and modern mathematical software, to calculate the absorption of microwaves in a sphere, as part of a study of possible effects of radar on human eyes. Both models are sophisticated and crude at the same time, albeit in different ways. Descartes ignored diffraction and polarization, whereas I include it - on the other hand, a homogeneous dielectric sphere far more faithfully represents a raindrop than an eye.

Philosophically, there seems little difference between what Descartes did three-and-a-half centuries ago and what I and many other physical scientists are doing today. The cultural difference is, however, enormous. Descartes' simulation was a substitute for an analytical solution that he was unable to find. But small computers are now so powerful, so easy to program, and so readily available that simulation has become routine in situations where there is no hope of encapsulating the phenomenon under investigation in a simple formula, and where the governing laws are known imprecisely, if at all. I have often thought that this change in scientific practice deserves serious and systematic study, so I approached this book with high expec-

The author surveys the application of computers for simulating our world and various imaginary ones - in a wide range of situations, including numerical weather forecasting, traffic flow in Albuquerque, New Mexico, and the evolution of artificial life inside a computer. Along the way, he discusses many subtle and important questions, such as when it is desirable for a model to be an accurate such accuracy might actually hinder the acquisition of insight and how complexity can emerge as large-scale behaviour in a system of many components that individually behave simply. tations. representation of external reality, when
I do have some criticisms. The book is disorganized, in the sense that many of the examples in chapters that ostensibly deal with different topics ("Pictures as programs", "The science of surprise", "Artificial worlds", "The reality of the virtual") could have been interchanged without the reader noticing. And recent research on the stability of the Solar System does not fit naturally in the lists of "surprise generators" or "nature's unanswered or unanswerable questions".

I was disappointed not to see any mention of quantum computers, whose different logic provides hope for the solution of problems that are uncomputable in the traditional way. A further criticism is that the colour plates are poorly reproduced and, in most cases, add little to their black-and-white duplicates in the text - in the traffic simulations, colour is important but almost impossible to see.

On the whole, the writing is lively and straightforward, although readers will wince at the phrase "it's place" and smile at the description of "nondeductive modes of reasoning - induction and abduction".

As a scholarly treatment of its subject, Casti's book fails. But as a popular account of the enormous change computers have brought to the everyday practice of science (Descartes notwithstanding), it is an easy and enjoyable read, and I can happily recommend it.

Michael Berry is at the H. H. Wills Physics Laboratory, University of Bristol, Tyndall Avenue, Bristol BS8 1TL, UK.
An account of the Hubble Space Telescope's troubled history and its importance in advancing our understanding of space introduces the photographs and clear explanations in Hubble's Universe: A New Picture of Space by Simon Goodwin. In this Hubble ultraviolet image of Saturn, the aurora at the north pole is clearly visible. Constable, $£ 14.95$.



